\title{
Diagnostics for a Magnetized Target Fusion Experiment
}

\author{
G. A. Wurden, T. P. Intrator, D. A. Clark, S. K. Coffey*, J. H. Degnan*, R. J. Maqueda, \\ E. L. Ruden*, J. M. Taccetti, and F. J. Wysocki \\ Los Alamos National Laboratory, Los Alamos, NM 87545 USA
}

*Air Force Research Laboratory, Kirtland Air Force Base, NM 87117 USA

\begin{abstract}
We are planning experiments using a Field Reversed Configuration (FRC) plasma injected into a metal cylinder, which is subsequently electrically imploded to achieve a fusing plasma. Diagnosing this plasma is quite challenging due to the short timescales, high energy densities, high magnetic fields, and difficult access. We outline our diagnostic sets in both a Phase I study (where the plasma will be formed and translated), and Phase II study (where the plasma will be imploded). The pre-compression plasma (diameter of only $8-10 \mathrm{~cm}$, length of $30-40 \mathrm{~cm}$ ) is expected to have $\mathrm{n} \sim 10^{17} \mathrm{~cm}^{-3}, \mathrm{~T} \sim 100$ $300 \mathrm{eV}, \mathrm{B} \sim 5 \mathrm{~T}$, and a lifetime of 10-20 microseconds. We will use visible laser interferometry across the plasma, along with a series of fiber-optically coupled visible light monitors to determine the plasma density and position. Excluded flux loops will be placed outside the quartz tube of the formation region, but inside of the diameter of the theta-pinch formation coils. Impurity emission in the visible and XUV range will be monitored spectroscopically, and fast bolometers will measure the total radiated power. A 20-Joule Thomson scattering laser beam will be introduced in the axial direction, and scattered light (from multiple spatial points) will be collected from the sides. Neutron diagnostics (activation and time-resolved scintillation detectors) will be fielded during both phases of the DD experiments.
\end{abstract}




\section{INTRODUCTION}

The goal of Magnetized Target Fusion (MTF) is to achieve a fusing plasma under conditions intermediate in both density and required power compared to standard Magnetic Fusion Energy (MFE) and Inertial Confinement Fusion (ICF) methods[1]. Our plan is to form, translate, and then rapidly compress (and thus heat) an FRC "target" plasma to fusion relevant conditions using an electrically-driven imploding solid metal liner[2].

The MTF plasma, both as initially formed, and also in its final state of compression, poses interesting diagnostic challenges. These challenges arise from limited access to the compact, high density, high magnetic field object of interest, as well as severe electromagnetic and mechanical force issues associated with the nearby imploding metal liner. A schematic of the FRC/Liner configuration is shown in Figure 1. The powerful and dynamic heating mechanism, namely stagnation of a rapidly moving solid metal liner and subsequent ohmic dissipation of a compact torus at 500 Tesla, should provide us with the opportunity to diagnose an extraordinary plasma. The diagnostic goal is to observe and characterize a $\beta \sim 1, \mathrm{n} \sim 10^{19} \mathrm{~cm}^{-3}$ deuterium, $\mathrm{T} \sim 10 \mathrm{keV}$ plasma, on timescales ranging down to 100 nanoseconds (formation time of $\sim 5-10$ microseconds, burn time of $\sim$ 1-2 microseconds).

\section{SPECIAL CONCERNS}

For an MTF experiment, there are three overall diagnostic sets that we must consider. The first, and simplest, involves diagnosing the initial (pre-compression) target plasma. 
Only after demonstrating the appropriate plasma density, temperature, lifetime, translation, and trapping, can the experiment logically proceed to the second (implosion) phase. During the implosion, another set of diagnostics is required to verify the symmetry and speed of the liner motion. Finally, a third set must be able to function during the 1-2 microsecond "burn phase", when the plasma is most highly compressed and fusing of the fuel (DD or even DT) is expected to occur. The phases and goals of the experimental program, along with corresponding diagnostics, are listed in Table 1. Access to the plasma, which is generally cylindrical in shape, is available end-on (axial), but very difficult to obtain side-on (radial). Side-on access is restricted first due to the close proximity of the segmented theta-pinch coils and the quartz tube, or after the plasma is translated, to the close fitting aluminum metal liner in the compression section. Furthermore, as the actual liner implosion begins, the plasma (and access) is rapidly reduced in size, and large electrical transients may be induced in any nearby electrical wiring or diagnostic circuits. Due to the incredibly high magnetic fields expected as a result of the liner compression (5 Tesla --> 500 Tesla), we also have the opportunity to field novel magnetic field sensors. Optical emission from the plasma will be variously affected by Stark, Zeeman or Paschen-Back, Doppler broadening, and self-absorption effects. There may be interfering emission from the cold outer layers of the plasma, especially on open field lines in the natural divertor region surrounding the FRC.

\section{INITIAL FRC DIAGNOSTICS}

The FRC plasma diagnostic set will consist of magnetics, spectroscopy, interferometry, bolometry, and Thomson scattering instruments. Diamagnetic flux loops [3] to measure 
excluded flux will be positioned underneath the theta pinch coils. The trick to this measurement is to be able to handle a large signal (which can be as big as the main bank voltage on the one-turn theta pinch coil)[4]. We will use visible light fiber/filter arrays oriented radially to observe the length of the FRC as it translates out of the formation region. These fibers can also be fed to spectrometers, which have gated/intensified detector arrays for spectral snapshots. However, due to significant Stark broadening (for example, $\sim 10 \mathrm{~nm}$ for $\mathrm{H}_{\beta}$ at $2 \times 10^{17} \mathrm{~cm}^{-3}$ ), spectra will be difficult to interpret. For observing XUV emission, we will have two systems. One will be standard silicon photodiode bolometry[5] to monitor total radiation, and the other will be a flat PET crystal spectrometer with a gated detector designed to look at Helium-like middle Z impurity lines (such as the $1.6 \mathrm{keV} 1 \mathrm{~s}^{2}-1 \mathrm{~s} 2 \mathrm{p}$ line from Aluminum, or perhaps Silicon). Both systems require vacuum access to the plasma, and as such will view axially from the ends of the machine (radial vacuum access through either the quartz tube or metal liner is too difficult or intrusive). Line averaged electron density will be obtained radially along at least one chord. An existing quadrature interferometer, operating in the blue at $442 \mathrm{~nm}$ with a 2-20 mW Linconix Helium-Cadmium laser is available for our use. A 90-degree Thomson scattering system to measure $\mathrm{n}_{\mathrm{e}}(\mathrm{r})$ and $\mathrm{T}_{\mathrm{e}}(\mathrm{r})$ at six spatial points is under construction. It has been carefully designed for unusually high dynamic range due to an expected high background level of plasma light, and utilizes a 16-bit PixelVision SpectraVideo 512 x 512 back-illuminated, cooled, 90\% quantum efficient CCD array detector, lens coupled to a Delft Instruments PP0370E gated image intensifier, with gain of up to 400,000 . Each CCD pixel is 24 microns square, and has a well depth of 350,000 electrons. A twin beam Quantel 20-nsec ruby laser provides $10 \mathrm{~J}$ per beam, in a 1-mm 
diameter waist inside the plasma. A total of 18 lines are imaged with a single holographic grating spectrometer (formerly used on the multi-point Thomson scattering system on the CTX spheromak at LANL[6]). Each spatial point has three measurements; one for the scattered light, and one adjacent above and below for plasma light subtraction. We are assuming that up to $90 \%$ of the detected light may be due to plasma backgrounds. As configured, the system should be capable of measuring electron temperatures between $10 \mathrm{eV}$ to $\sim 350 \mathrm{eV}$.

\section{LINER DIAGNOSTICS}

In 1999, we conducted two aluminum liner vacuum test shots to check liner stability and velocity on the Shiva-Star capacitor facility at Phillips Laboratory in Albuquerque[7]. Diagnostics included multi-time x-ray radiographic images of the metal liner, internal magnetic field probe measurements to monitor compression of the liner as a function of time by measuring the increase in field strength of a seed magnetic field, and optical "pin" fibers which monitor liner symmetry by observing the arrival time of the inner surface of the liner at pre-determined points. There was good agreement between the diagnostics (described below) that measure this total magnetic field, the time of impact data, the radiograph images, and analytic model expectations. With the possible exception of the $\mathrm{x}$-ray radiography (which is not detailed here), none of these diagnostics will carry over into the later integrated plasma/liner tests.

Since these test shots had no plasma, we installed two central diagnostic stalks, one from above, and one from below the liner, in positions which would be filled with an FRC 
plasma in a "real" shot, as shown in Figure 2. A thin aluminum cylindrical liner is mounted between upper and lower "glide planes". The glide plane refers to thick, immobile metal end-pieces along which the liner "glides" when being accelerated by JxB forces. Optical pin fibers in the upper stalk measured the liner time of impact via shock induced fluorescence in the fibers. The stalk diameter near the "pins" could not be any smaller than $1.0 \mathrm{~cm}$ because the optical fibers were already at their minimum bend radius when installed in the stalk. The liner stopped here at a 9.8:1 radial compression ratio. The bottom $0.635 \mathrm{~cm}$ diameter stalk included arrays of magnetic loop (B-dot) probes, and allowed the liner to travel up to a 15.4:1 radial compression ratio. Through the center of both stalks we routed a polarization preserving optical fiber for a novel ZnSe Faraday rotation sensor. The two stalks were joined at a concentric bushing. Permanent magnets (shown in Figure 2) were used for a seed magnetic field that varied from 60-90 Gauss throughout the interior of the liner region of interest. The axial and radial components of the initial seed magnetic field were measured with a LakeShore Cyrogenics Hall Probe, and modeled with a magnetostatic code (FLUX2D). Measurements of the evolving initial magnetic field with B-dot and Faraday techniques provided a way determine the time history of the liner radius.

\section{A. Pin detector}

The pin detector[8] is a glass $50 \mu \mathrm{m}$ fiber that emits a burst of Cerenkov radiation when it is shocked on the end by the arrival impact of the liner. This furnishes a precise measurement of the time of impact, with a time resolution in this application of 20$50 \mathrm{nsec}$. The sharp rise time of the Cerenkov signal is usually unambiguous unless there is a temporally smeared shock front, as was the case for our pin detectors installed near 
the glide plane. The optical signal was routed into the screen room control center via 60 meters of fiber, and then converted to electrical signals with Optelecom transmitters. These were amplitude calibrated to each other, and time synchronized for trigger and group delay. Eighteen electrical signals in the screen room were digitized on VXI Tektronix TEK641 recorders at 4 nsec per sample, with data records of 8k samples, 8 bits deep.

\section{B. B-dot probe array}

The B-dot axial probe array was shielded electrostatically inside a 1/4" SS tube, with 0.010" walls. At each of five axial locations spaced from the bottom glide plane up to the mid-plane, there were axial $B_{z}$ and radial $B_{r} B$-dot coils. These were overlaid in wound/counterwound pairs to give us differential signals so we could take advantage of common mode rejection by taking sums and differences of the signals. Typical data has $\mathrm{B}_{\mathrm{z}}$ and significant $\mathrm{B}_{\mathrm{r}}$, and canceling derivatives from the convective derivative part of the time derivative due to the moving liner reference frame for the liner image currents.

The shape of liner was inferred from B-dot array data. The SS tube around B-dot probe adds attenuation and time delay as magnetic field soaks through cylindrical wall, so we had to deconvolve its response using spectral methods.

\section{Faraday optical probe}

The novel Faraday sensor used a high Verdet constant ZnSe crystal to measure the magnetic fields. It's advantage is that it does not have to deal with electrical noise or pickup due to the optical nature of the signal. A He-Ne pump laser provided polarized light whose polarization was rotated as the magnetic field at the crystal increased. The range of this diagnostic stretched from approximately 400-10000 Gauss, but in fact could 
easily measure much larger fields with the same sensitivity and noise floor. The active crystal was sandwiched between two polarization preserving optical fibers, polarizer splitter, and GRIN lenses. The polarization locked fibers thread the axis, and need to be brought out of the vacuum can via epoxy seals to the screen room where the pump laser was situated.

\section{INTEGRATED TEST OF PLASMA COMPRESSION}

In the future, during integrated plasma/liner/implosion experiments, when the plasma and magnetic fields are compressed by the aluminum metal liner over a period of $10-20$ microseconds, the shot repetition rate will be reduced from 10-20 per day (without implosion) to only once per week. The liner and nearby structures will be destroyed or damaged from the multi-MJ implosion. Due to the structures around the liner, which include both guide field magnetic coils and the return leg of the liner electrical feeds, as well as $\mathrm{HV}$ electrical insulation (see Figure 1), we believe it will be extraordinarily difficult to have side-on access during the implosion phase. Even end-on optical access will eventually become restricted when the ends of the nominally cylindrical liner impact the axis of symmetry, creating high velocity molten metal jets that squirt out the ends of the experiment. It will still be useful to observe x-ray lines of selected impurities (such as Helium-like Aluminum or Silicon), and their corresponding broadening due to Stark and strong magnetic field splitting (Paschen-Back) effects. We may be able to spectroscopically study impurity contamination issues by coating the inner wall of the aluminum liner with selected materials[9]. Of course, neutrons will get out, and we intend to field both time resolved (scintillating plastic/PMT) and total neutron yield (activation) measurements. Prior to compression, the neutron signal will be small but detectable due 
to the high plasma density. Then, as the density increases a factor of $100 \mathrm{x}$ and the temperature increases a factor of 10-20x, the neutron time history and absolute levels will be essential for judging the success of the implosion, relative to code modeling.

\section{ACKNOWLEDGEMENTS}

This work is supported at LANL by the US DOE Office of Fusion Energy Sciences and LANL LDRD through contract \#W-7405-ENG-36. Joint experiments have been, and will be performed in collaboration with the Air Force Research Laboratory in Albuquerque, New Mexico.

\section{REFERENCES}

1. R. E. Siemon, I.R. Lindemuth, and K.F. Schoenberg, Comments Plasma Physics and Controlled Fusion, 1999. 18(6): p. 363-386.

2. Glen A. Wurden, Kurt F. Schoenberg, Richard E. Siemon, Michel Tuszewski, Fred J. Wysocki, Richard D. Milroy, Journal Plasma Fusion Research SERIES vol. 2,. 1999: Toki, Japan. p. 238-241.

3. M. Tuszewski, Phys. Fluids, 1981. 24(11): p. 2126-2127.

4. M. Tuszewski and W.T. Armstrong, Rev. Sci. Instrum., 1983. 54(12): p. 16111614.

5. R. J. Maqueda, G. A. Wurden, and E. A. Crawford, Rev. Sci. Instrum., 1992. 63(10/pt.2): p. 4717-4719. 
6. Rita Gribble, T. R. Jarboe, H. W. Hoida, J. Lipson, E. W. Newman, Technical Report LA-105280-MS, Sept. 1985, Los Alamos National Laboratory: Los Alamos, NM.

7. R. E. Siemon, T. P. Intrator, M. J. Taccetti, F. J. Wysocki, R. J. Faehl, K. F. Schoenberg, D. A. Clark, K. Forman, I. R. Lindemuth, G. A. Wurden, MTF Team (LANL), J. H. Degnan, D. Gale, W. Sommars, S. K. Coffey, Proceedings of the 18th International Conference on Plasma Physics and Controlled Nuclear Fusion Research. 2000. Sorrento, Italy. To be published.

8. L. R. Veeser, M. J. George, R. Menikoff, B. Papatheofanis, J. S. McGurn, P. L. Gobby, Fiber Optic and Laser Sensor V. 1987, 838 pg. 60-68: (1988). Society of Photo-Optical Instrumentation Engineers.

9. J. H. Degnan, R. Bartlett, T. Cavazos, D. Clark, S. K. Coffey, R. J. Faehl, M. Frese, D. Fulton, D. Gale, T. W. Hussey, et al., 3rd Symposium on Current Trends in International Fusion Research. 1999. Washington, D. C.: NRC Research Press. To be published. 


\section{CAPTIONS}

Figure 1. Cut-away schematic of FRC plasma injector mated to liner implosion driver. The plasma is first formed in the quartz tube under the theta-pinch coil, and then translated into the $1 \mathrm{~mm}$ thick aluminum liner, which is then imploded by a multi-MA current pulse, to heat the plasma to fusion relevant parameters.

Figure 2. Cross section of the 10-cm diameter, 30-cm tall , 1-mm thick aluminum liner, and central diagnostic stalk used to study the electrically driven implosion, without plasma present. The permanent magnets provided a seed magnetic field for diagnostic purposes. The upper stalk contained the pin fibers and $\mathrm{ZnSe}$ Faraday diagnostic. The lower stalk contained the B-dot probes.

Table 1. Summary of experimental research goals and requirements for FRCbased MTF. 


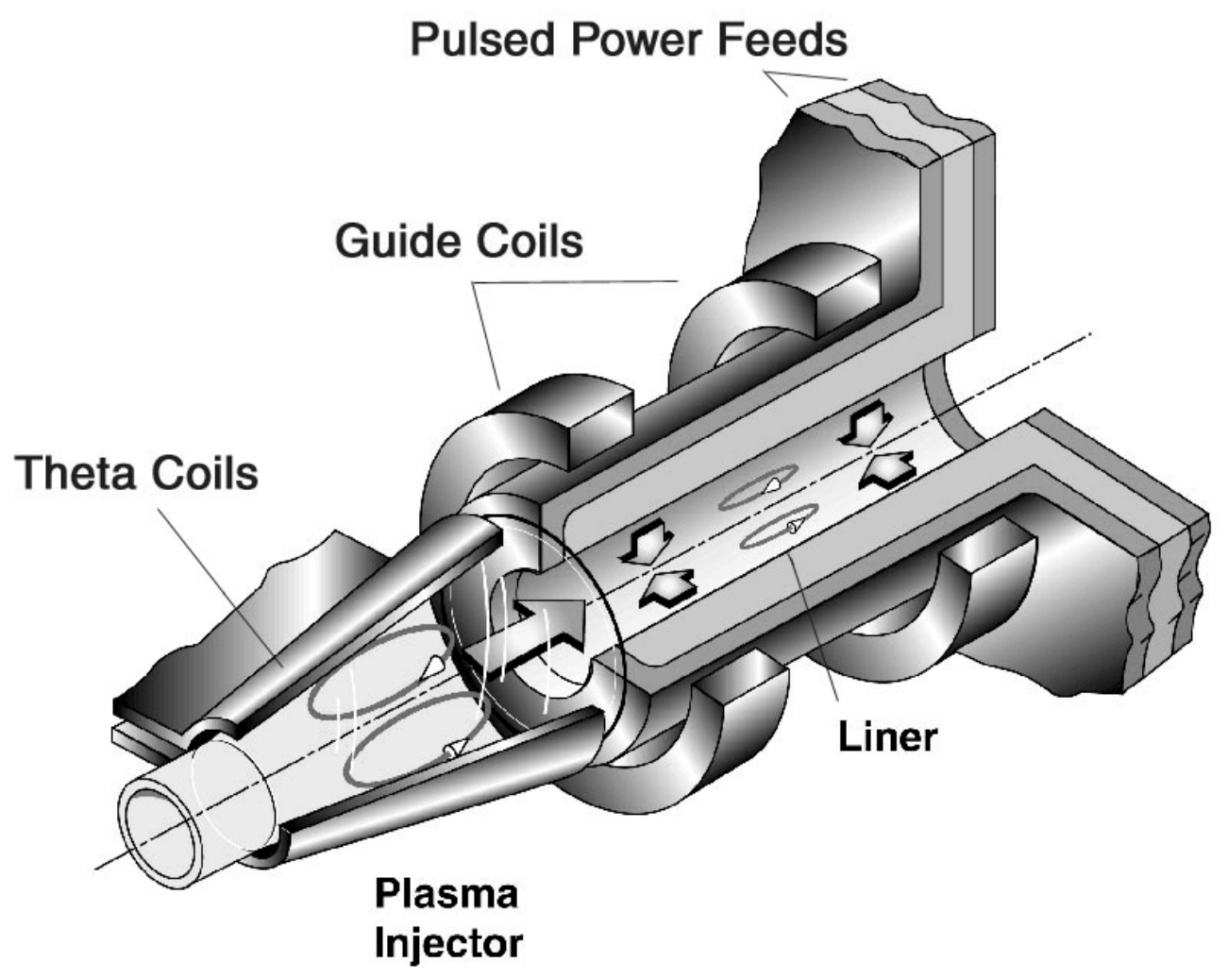

Figure 1

Wurden, et. al.

HTPD

BP23 


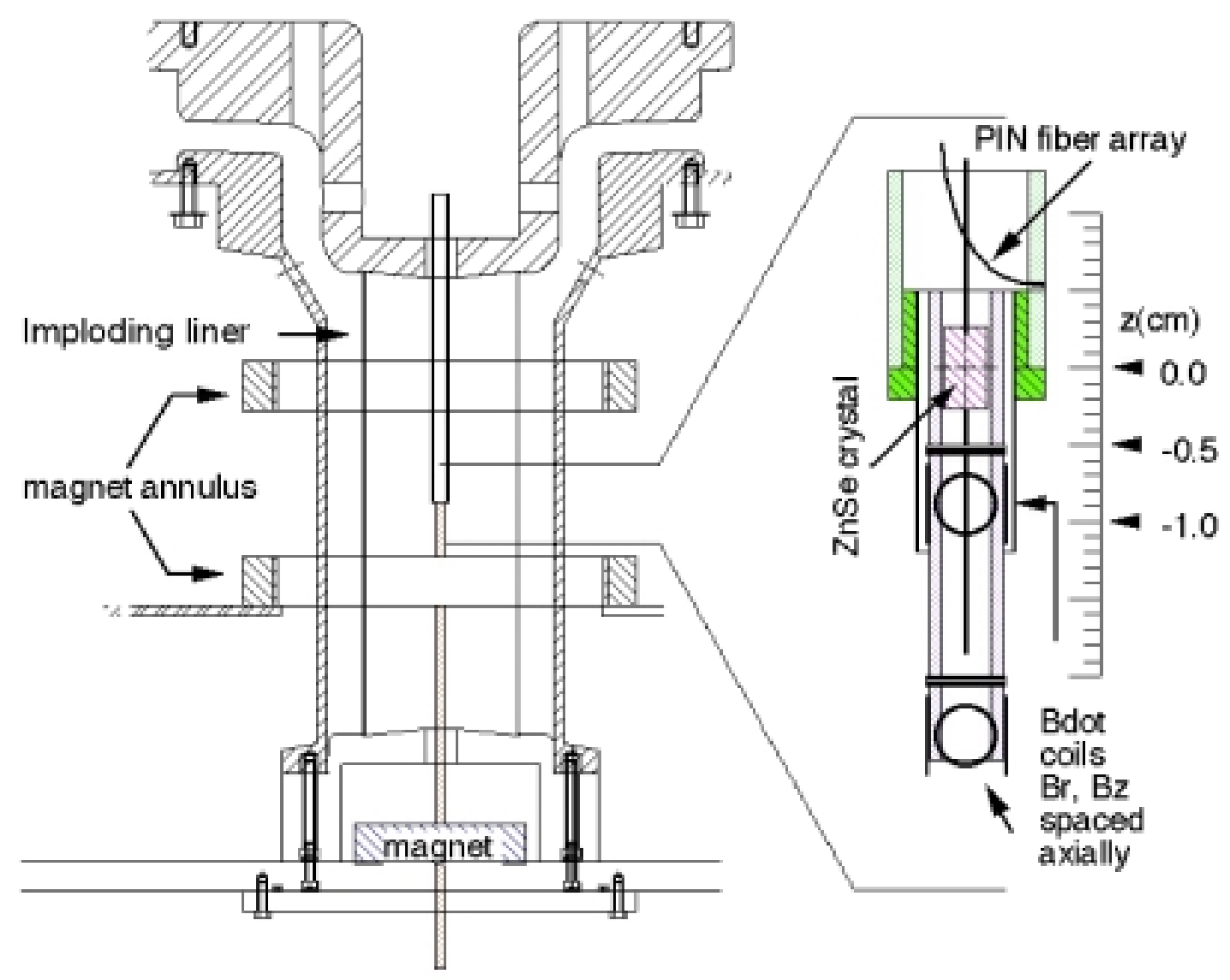

Figure 2

Wurden, et. al.

HTPD

BP23 


\begin{tabular}{|c|c|c|c|}
\hline Research Goals & Scientific/Technical Issues & $\begin{array}{l}\text { Principal } \\
\text { Facility }\end{array}$ & Principal Diagnostics \\
\hline $\begin{array}{l}\text { Vacuum liner compression } \\
\text { from } r_{i}=5 \mathrm{~cm} \text { to } r_{f}=0.5 \mathrm{~cm} \\
\text { with } V>0.3 \mathrm{~cm} / \mu \mathrm{s}\end{array}$ & $\begin{array}{l}\text { Rayleigh-Taylor and kink } \\
\text { stability for } \mathrm{L} / \mathrm{D} \sim 3, \\
\text { Convergence } \mathrm{R}_{\mathrm{i}} / \mathrm{R}_{\mathrm{f}} \sim 10 \\
\text { * completed FY99 }\end{array}$ & $\begin{array}{l}\text { Shiva-Star } \\
\text { (AFRL) }\end{array}$ & $\begin{array}{l}\text { Side-on radiography } \\
\text { Magnetics probes } \\
\text { Faraday probe } \\
\text { Pin arrays }\end{array}$ \\
\hline $\begin{array}{lr}\text { FRC } & \text { formation } \\
\text { Suitable } & \text { for } \\
\text { Compression } & \\
\mathrm{T} \sim 300 \mathrm{eV} & \\
\mathrm{n} \sim 10^{17} \mathrm{~cm}^{-3} & \\
\tau_{\mathrm{E}}>10 \mu \mathrm{s} & \end{array}$ & $\begin{array}{l}\text { FRC formation at high density } \\
\text { Stability for } S^{*} / \mathrm{E}<3.5 \\
\text { Confinement } \tau_{\mathrm{E}} \sim 0.5 \mathrm{R}^{2} / \rho_{\mathrm{i}} \\
\text { Impurity Content } \mathrm{Z}_{\mathrm{eff}}<2 \\
\text { *under construction } \mathrm{FY} 00\end{array}$ & $\begin{array}{l}\text { Colt } \\
\text { (LANL) }\end{array}$ & $\begin{array}{l}\text { Excluded flux } \\
\text { B probe array } \\
\text { Interferometry } \\
\text { Thomson scattering } \\
\text { Bolometry } \\
\text { Optical spectroscopy }\end{array}$ \\
\hline $\begin{array}{l}\text { FRC Translation and } \\
\text { trapping into a liner with } \\
r_{\text {wall }}=5 \mathrm{~cm}, \\
r_{\mathrm{s}} \sim 3 \mathrm{~cm} \\
1_{\mathrm{s}}=30 \mathrm{~cm}\end{array}$ & $\begin{array}{l}\text { Maintaining stability } \\
\text { Impurity content }\end{array}$ & $\begin{array}{l}\text { Colt } \\
\text { (LANL) }\end{array}$ & $\begin{array}{l}\text { B probe array } \\
\text { Bolometry } \\
\text { Interferometry } \\
\text { Spectroscopy } \\
\text { Neutron (test) }\end{array}$ \\
\hline $\begin{array}{l}\text { Integrated Compression of } \\
\text { FRC in liner to T } \sim 5 \mathrm{keV} \\
\mathrm{n} \sim 10^{19} \mathrm{~cm}^{-3} \tau_{\mathrm{E}} \sim 1 \mu \mathrm{s}\end{array}$ & $\begin{array}{l}\text { Stability } \\
\text { Transport } \\
\text { Impurity content (liner mix) }\end{array}$ & $\begin{array}{l}\text { Shiva-Star } \\
\text { (AFRL) }\end{array}$ & $\begin{array}{l}3 \text { axis radiography } \\
\text { End-on interferometry } \\
\text { Spectroscopy } \\
\text { Bolometry } \\
\text { Neutron }\end{array}$ \\
\hline
\end{tabular}

